\title{
Early Run 2 Hard QCD Results from the ATLAS Collaboration
}

\author{
Nicola Orlando ${ }^{1,2, a}$ on behalf of the ATLAS Collaboration \\ ${ }^{1}$ The University of Hong Kong, Department of Physics, Hong Kong \\ ${ }^{2}$ Aristotle University of Thessaloniki, Laboratory of Nuclear and Particle Physics, Thessaloniki, Greece, \\ 54124
}

\begin{abstract}
We provide an overview of hard QCD results based on data collected with the ATLAS detector in proton-proton collision at $\sqrt{s}=13 \mathrm{TeV}$ at the Large Hadron Collider. The production of high transverse momentum jets, photons and photon-pairs were studied; the inclusive jet cross section is found to agree well with the prediction of perturbative QCD calculations performed at next-to-leading accuracy. The production cross sections for $\mathrm{W}$ and $\mathrm{Z}$ bosons in their $e$ and $\mu$ decays was measured; in general, agreement is found with the expectation of next-to-next-to leading order QCD calculations and interesting sensitivities to the proton structure functions are already observed. The top production cross sections were measured in different top decay channels and found to agree with the state of the art QCD predictions.
\end{abstract}

\section{Introduction}

Since June 2015 the Large Hadron Collider (LHC) has started to deliver proton-proton (pp) collisions at the unprecedented centre-of-mass energy $\sqrt{s}=13 \mathrm{TeV}$. The ATLAS [1] collaboration has analysed this very early $13 \mathrm{TeV}$ data taken in both special conditions with very low pileup and those dedicated to high $p_{\mathrm{T}}$ physics.

The $13 \mathrm{TeV}$ ATLAS physics program benefits from the installment of the Insertable B-Layer (IBL) [2], a fourth layer added to the present Pixel detector between a new beam pipe and the current inner Pixel layer (B-layer) and many other improvements affecting the detector, data-taking as well as analysis software-framework.

The measurements presented in this paper use between $6.4 \mathrm{pb}^{-1}$ up to $85 \mathrm{pb}^{-1}$ of integrated luminosity collected at LHC in proton-proton (pp) collisions at $\sqrt{s}=13 \mathrm{TeV}$. The precision of many measurements is limited by the early luminosity calibration is based on pair of $\mathrm{x}-\mathrm{y}$ beam-separation scans performed in June 2015, leading to about $9 \%$ uncertainty on the luminosity scale determination.

\section{Jets and photons measurements}

The measurement of cross-sections of jets produced at high transverse momentum provides a test of the validity of perturbative QCD (pQCD) at the TeV scale.

\footnotetext{
a e-mail: nicola.orlando@cern.ch
} 


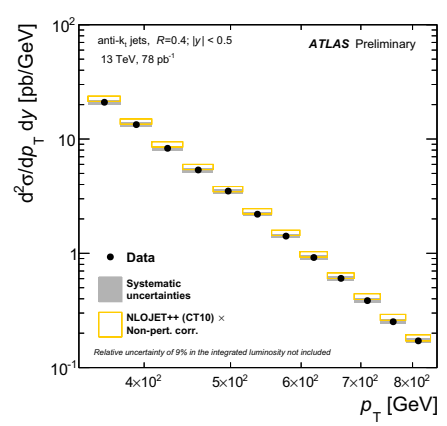

(a)

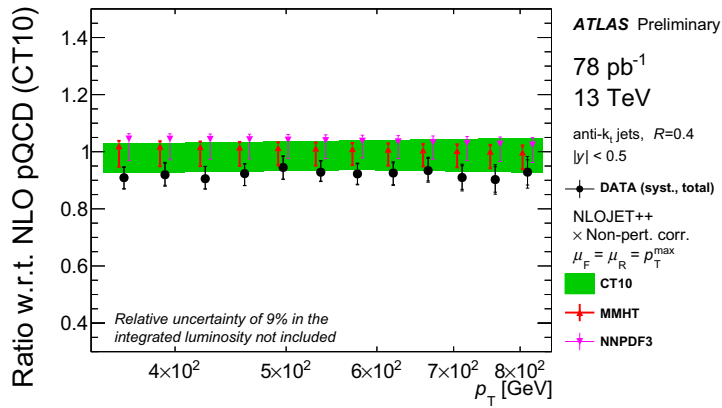

(b)

Figure 1. Inclusive-jet cross sections (a) as a function of the jet $p_{\mathrm{T}}$ in $|y|<0.5$; the error bars show the statistical uncertainties and the filled area shows the experimental systematic uncertainties. Ratio of the measured inclusivejet cross section to the NLO pQCD prediction (b), shown as a function of the jet $p_{\mathrm{T}}$ in $|y|<0.5$. The figures are taken from Ref. [3].

The inclusive-jet cross section is measured differentially as a function of the jet transversemomentum, $p_{\mathrm{T}}$, in a jet rapidity range of $|y|<0.5[3]^{1}$. Theoretical predictions at next-to-leading order (NLO) in pQCD are available from the NLOJET++ generator [4]; the predictions are corrected for non-perturbative effects. As shown in Fig. 1, the pQCD calculations are consistent with the measurement; the $9 \%$ uncertainty on the luminosity calibration is not included in the figures.

The production of prompt photons provides a test for $\mathrm{PQCD}$ in a cleaner environment compared to jet production, since it is less affected by hadronisation effects. The measurement of two prompt isolated photons is a tool to probe $\mathrm{pQCD}$ predictions relevant to understand the irreducible background to physics processes involving photons in the final state, like the Higgs boson decay in photon-pair.

The kinematic distributions of the signal yield for inclusive photon production [5], presented as functions of photon transverse-energy, $E_{\mathrm{T}}^{\gamma}$, and pseudorapidity, $\left|\eta^{\gamma}\right|$, agree with the predictions of the SHERPA generator [6], as shown in Fig. 2 (a-b).

Fig. 3 shows the invariant mass spectrum, $m_{\gamma \gamma}$, and the transverse-momentum distribution, $p_{\mathrm{T}}^{\gamma \gamma}$, of the selected photon-pairs [7]. An enhancement in the $p_{\mathrm{T}}^{\gamma \gamma}$ spectrum is observed between $20 \mathrm{GeV}$ and $40 \mathrm{GeV}$, about the sum of the $E_{\mathrm{T}}^{\gamma}$ thresholds of the two photons, as expected from beyond leading-order (LO) QCD corrections.

\section{Heavy flavour production}

The study of heavy quarkonium is a challenge for the understanding of perturbative and nonperturbative QCD regimes. Although many aspects of quarkonium production and decay can be described reasonably well, the literature lack of a coherent theoretical picture which can explain all quarkonium measurements simultaneously. A first analysis of the non-prompt fraction of $J / \psi$ production at $\sqrt{s}=13 \mathrm{TeV}$ is performed in the di-muon decay channel [8]. The non-prompt fraction is found

\footnotetext{
${ }^{1}$ ATLAS uses a right-handed coordinate system with its origin at the nominal interaction point (IP) in the centre of the detector and the $\mathrm{z}$-axis along the beam pipe. The $\mathrm{x}$-axis points from the IP to the centre of the LHC ring, and the $\mathrm{y}$-axis points upward. Cylindrical coordinates $(r, \varphi)$ are used in the transverse plane, $\varphi$ being the azimuthal angle around the beam pipe. The pseudorapidity is defined in terms of the polar angle $\theta$ as $\eta=-\ln [\tan (\theta / 2)]$. Transverse momentum and energy are defined as $p_{\mathrm{T}}=p \sin \theta$ and $E_{\mathrm{T}}=E \sin \theta$, respectively.
} 


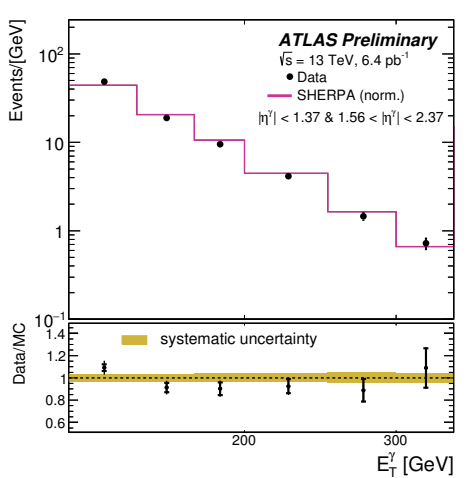

(a)

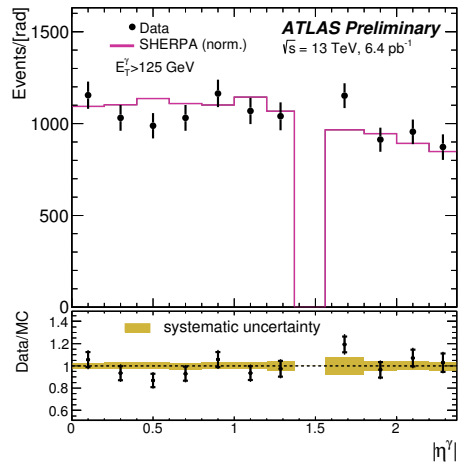

(b)

Figure 2. Measured isolated-photon yield as a function of $E_{\mathrm{T}}^{\gamma}$ (a) and $\left|\eta^{\gamma}\right|$ (b) for $\left|\eta^{\gamma}\right|<2.47$. The figures are taken from Ref. [5].

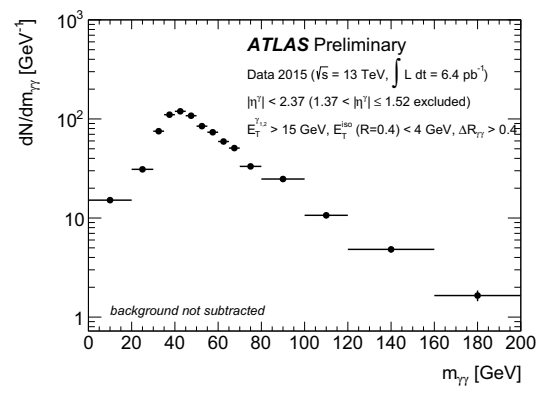

(a)

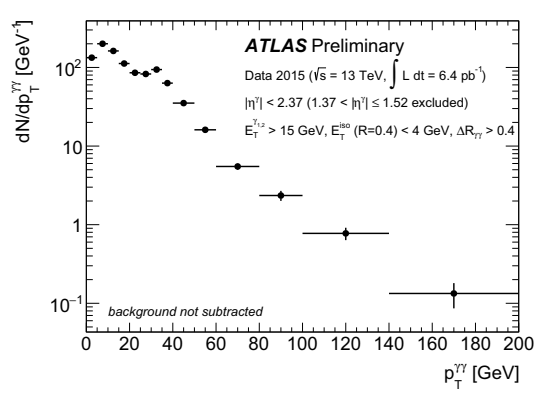

(b)

Figure 3. Number of isolated photon-pair candidates shown as a function of $m_{\gamma \gamma}$ (a) and $p_{\mathrm{T}}^{\gamma \gamma}$ (b). The figures are taken from Ref. [7].

to increase steadily from 0.25 at the $J / \psi$ transverse momentum of $8 \mathrm{GeV}$ to 0.65 at $40 \mathrm{GeV}$, with no significant variation with rapidity observed within the precision of the measurement. The centre-ofmass energy (and initial-state) dependence of the non-prompt fraction is studied by comparing these results to previous ATLAS measurements at $\sqrt{s}=2.76 \mathrm{TeV}$ and at $\sqrt{s}=7 \mathrm{TeV}$, and to CDF measurements $\sqrt{s}=1.96 \mathrm{TeV}$. The comparison between the various measurements is illustrated in Fig. 4; no significant change in the non-prompt fraction is observed from $\sqrt{s}=7 \mathrm{TeV}$ to $\sqrt{s}=13 \mathrm{TeV}$, contrary to the significant difference observed between the $\sqrt{s}=7 \mathrm{TeV}$ measurement and the measurements at lower energies.

\section{$4 \mathrm{~W} / \mathrm{Z}$ cross sections}

The measurement of weak vector bosons, $\mathrm{W} / \mathrm{Z}$, production at hadron colliders provides a benchmark of our understanding of QCD and electroweak (EW) phenomenology. The large production cross 


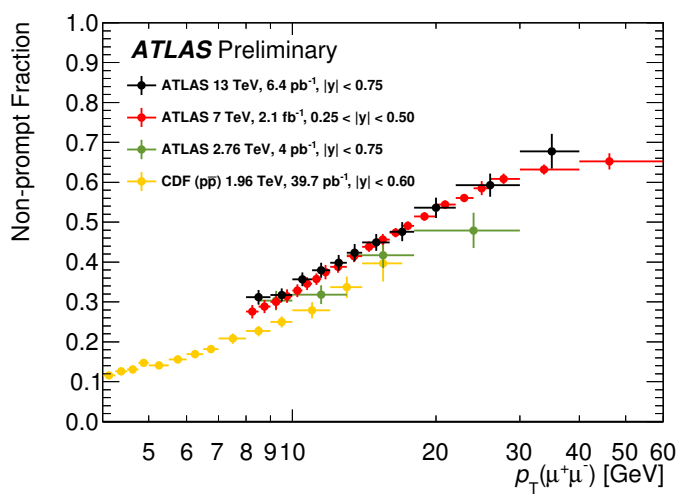

Figure 4. Non-prompt $J / \psi$ production fraction measured in the rapidity $|y|<0.75$ compared to previous measurements from ATLAS in pp collisions at $\sqrt{s}=2.76 \mathrm{TeV}$ and $\sqrt{s}=7 \mathrm{TeV}$, and from CDF in $p \bar{p}$ collisions at $\sqrt{s}=1.96 \mathrm{TeV}$. The error bars represent the total uncertainty on the measurements (statistical and systematic). The figures are taken from Ref [8].

sections and easily identifiable $\mathrm{W} / \mathrm{Z}$ decays to leptonic final states offer a clean experimental signature which can be measured to high precision. In addition, theoretical predictions for inclusive $\mathrm{W} / \mathrm{Z}$ production are available at next-to-next-to-leading-order (NNLO) accuracy in QCD, and include EW corrections at NLO accuracy [9]; the cross-section predictions are dependent on the parton distribution functions (PDFs), therefore, measurements of $\mathrm{W}$ and $\mathrm{Z}$ bosons production offer a unique opportunity to test modeling of PDFs at the new centre-of-mass energy of $\sqrt{s}=13 \mathrm{TeV}$.

Fig. 5 (a-b) shows the measured fiducial W/Z cross sections [10]. The data are compared to predictions calculated using different PDF sets. The measurements agree well with the predictions. The experimental precision is already comparable to PDF uncertainties; however, the current experimental precision is limited by the preliminary luminosity calibration.

The measurement of the ratio $R_{W^{+} / W^{-}}=\sigma_{W^{+}}^{f i d} / \sigma_{W^{-}}^{f i d}[10]$ is shown in Fig 5 (c); there is a significant scatter for different PDF predictions and the accuracy of the experimental result is comparable to the spread among them. The data favours results from the PDFs which include LHC measurements lower centre-of-mass energy (NNPDF3.0 [11], MMHT14NNLo68cL [12], and ABM12LHC [13]).

Measurements of vector bosons production in association with jets represent an important test of pQCD and constitute a relevant background for studies of the Higgs boson and searches for new phenomena. The fiducial production cross sections for $\mathrm{Z}$ bosons plus inclusive one to four jet multiplicities have been measured [15] with a precision ranging from about $\sim 10 \%$ to $\sim 20 \%$. The measurements have been compared to predictions from SHERPA and MADGRAPH [16], normalised to the NNLO inclusive cross-section for $\mathrm{Z}$ boson production; as can be observed from Fig. 6, the predictions show a reasonable agreement with the data.

\section{Top quark pair cross sections}

The top quark is the heaviest known elementary particle, with a mass close to the scale of electroweak symmetry breaking. Precise measurements of the top-pair cross section, $\sigma_{\bar{t}}$, are sensitive to the gluon parton distribution function (PDF) of the proton, the strong coupling constant $\alpha_{s}$, the top-quark mass $m_{t}$, and potential enhancements due to physics beyond the Standard Model (SM). 


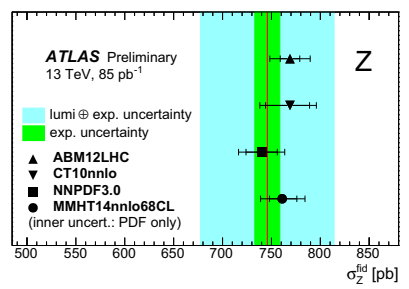

(a)

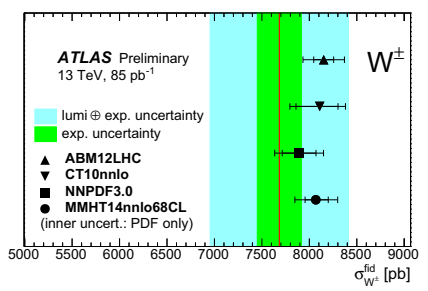

(b)

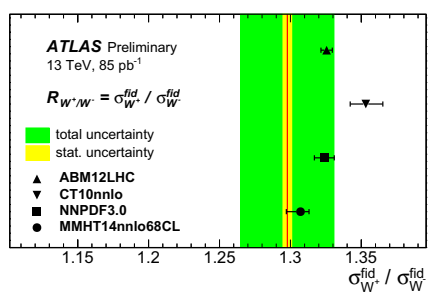

(c)

Figure 5. Measured fiducial W/Z cross sections (a,b) and $R_{W^{+} / W^{-}}$(c) (red line); the green (cyan) band corresponds to the experimental uncertainty without (with) the luminosity uncertainty; the yellow band in (c) represent the statistical uncertainty on the measurement. The data are compared to NNLO QCD predictions derived with four PDF sets; the inner error bar of the predictions represents the PDF uncertainty while the outer error bar includes the sum of all other systematics. The figures are taken from Ref [10].
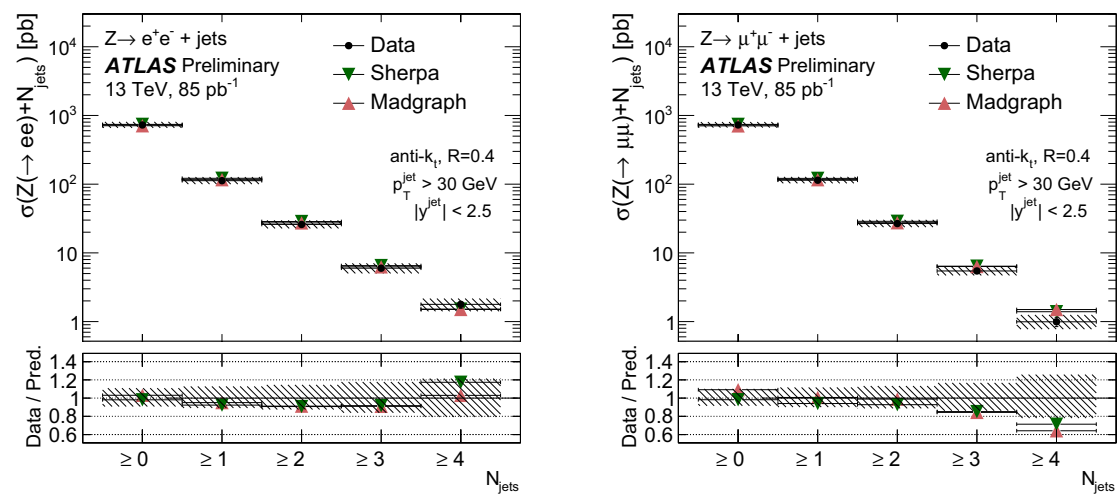

Figure 6. Fiducial cross sections as a function of the inclusive jet multiplicity in the $\mathrm{Z} \rightarrow e e$ channel (left) and the $\mathrm{Z} \rightarrow \mu \mu$ channel (right). The measured cross section is compared to predictions from SHERPA and MADGRAPH. The hatched error band corresponds to the total systematic and statistical uncertainty of the cross section, together with the luminosity uncertainty. The figures are taken from Ref [15].

The inclusive $t \bar{t}$ production cross-section was measured in several decay channels, assuming $m_{t}=$ 175.5 GeV [17, 18]. As shown in Fig. 7 (a), the data agree well with the state of the art SM prediction based on NNLO QCD perturbative expansion supplemented with next-to-next-to-leading logarithm (NNLL) QCD resummation [19].

A measurement of the ratio between the $\bar{t}$ and Z-boson production cross-sections is also presented, using on the measurement of the $\bar{t}$ cross-section in the $e \mu$ final state and of the Z-boson cross-section in the $e e$ and $\mu \mu$ decay modes. The measurement of the $t \bar{t}$ cross-section in the $e \mu$ final state is used to calculate the ratio since it is the most precise ATLAS measurement of the $t \bar{t}$ cross-section at $\sqrt{s}=$ $13 \mathrm{TeV}$. This ratio benefits from significant cancellations in systematic uncertainties between the two cross-section measurements, and is sensitive to ratios of the gluon and sea-quark PDFs. 

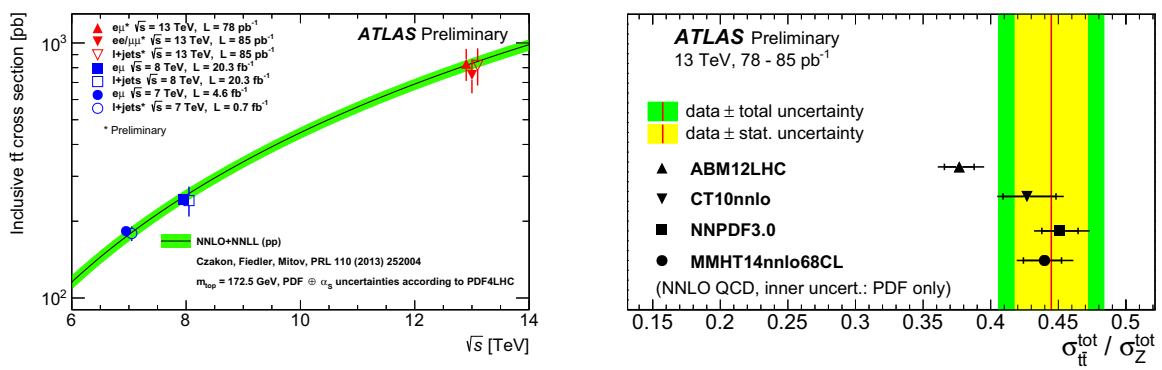

Figure 7. Cross-section for $t \bar{t}$ pair production in pp collisions as a function of centre-of-mass energy (a); the ATLAS measurements at $\sqrt{s}=7,8$ and $13 \mathrm{TeV}$ are compared to the NNLO+NNLL theory predictions. Measured cross-section ratio $R_{\bar{t} / \mathrm{Z}}$ compared to NNLO predictions at $\sqrt{s}=13 \mathrm{TeV}$ based on several PDF sets; the inner shaded band corresponds to the statistical uncertainty on the measurement, whilst the outer shaded band includes both statistical and systematic uncertainties; the inner error bars on the predictions correspond to PDF uncertainties only, while the outer error bars also include QCD scales and $\alpha_{s}$ uncertainties. The figures are taken from Ref $[17,18]$.

The measured $\bar{t} / \mathrm{Z}$ cross-section ratio, $R_{\bar{t} / \mathrm{Z}}[18]$, agree with predictions obtained with CT10NNLO, NNPDF3.0 and MMHT14NNL068CL, as can be seen from Fig. 7 (b). However, data are only marginally consistent with the prediction derived with the ABM12LHC PDF set, which gives a 12\% smaller $\bar{t} \bar{t}$ cross-section than CT10NNLO.

\section{References}

[1] ATLAS Collaboration, JINST 3 (2008) S08003.

[2] ATLAS collaboration, CERN-LHCC-2010-013. ATLAS-TDR-19 (2010).

[3] ATLAS collaboration, ATLAS-CONF-2015-034 (2015).

[4] Z. Nagy, Phys. Rev. D 68 (2003) 094002 [hep-ph/0307268].

[5] ATLAS collaboration, ATL-PHYS-PUB-2015-016 (2015).

[6] T. Gleisberg et al. JHEP 0902 (2009) 007 [arXiv:0811.4622 [hep-ph]].

[7] ATLAS collaboration, ATL-PHYS-PUB-2015-020 (2015).

[8] ATLAS collaboration, ATLAS-CONF-2015-030 (2015).

[9] Y. Li and F. Petriello, Phys. Rev. D 86 (2012) 094034 [arXiv:1208.5967 [hep-ph]].

[10] ATLAS collaboration, ATLAS-CONF-2015-039 (2015).

[11] R. D. Ball et al. [NNPDF Collaboration], JHEP 1504 (2015) 040 [arXiv:1410.8849 [hep-ph]].

[12] L. A. Harland-Lang et al. Eur. Phys. J. C 75 (2015) 5, 204 [arXiv:1412.3989 [hep-ph]].

[13] S. Alekhin et al. Phys. Rev. D 89 (2014) 5, 054028 [arXiv:1310.3059 [hep-ph]].

[14] J. Gao et al., Phys. Rev. D 89 (2014) 3, 033009 [arXiv:1302.6246 [hep-ph]].

[15] ATLAS collaboration, ATLAS-CONF-2015-041 (2015).

[16] J. Alwall et al., JHEP 1407 (2014) 079 [arXiv:1405.0301 [hep-ph]].

[17] ATLAS collaboration, ATLAS-CONF-2015-033 (2015).

[18] ATLAS collaboration, ATLAS-CONF-2015-049 (2015).

[19] M. Czakon and A. Mitov, Comput. Phys. Commun. 185 (2014) 2930 [arXiv:1112.5675 [hep$\mathrm{ph}]$. 